\title{
Dither Shape in the Averaging of Switched Systems
}

\author{
Luigi Iannelli Karl Henrik Johansson Ulf T. Jönsson Francesco Vasca
}

\begin{abstract}
It was shown by Zames and Shneydor that a high-frequency dither of a quite arbitrary shape can be used to smooth the effective nonlinear sector of Lipschitz continuous feedback systems. Here it is shown that also systems with discontinuous nonlinearities can be smoothed using dither signals, as long as the amplitude distribution function of the dither is Lipschitz continuous.
\end{abstract}

\section{INTRODUCTION}

A frequently used technique to stabilize a nonlinear feedback system in Lur'e form is by injecting a high-frequency dither signal to decrease the effective nonlinear sector. If the dither frequency is sufficiently high, the dithered system behaves qualitatively the same as an averaged system, in which the dither and the nonlinearity are replaced by another nonlinearity. Control design is then done for the averaged system, but applied to the dithered. For Lipschitz continuous systems this scheme can be justified rigorously by using classical averaging theory [1]. In [2], [3], Zames and Shneydor discussed how the behavior of the system depends on the amplitude distribution function of the dither for the case when the nonlinearities are Lipschitz continuous. For systems with nonsmooth nonlinearities there are only rigorous treatments of systems with particular nonlinearities and dithers, such as pulse-width modulated systems [4], [5], power converters [6], and relay systems [7], [8]. The current paper provides an averaging theorem for a general class of switched systems with a quite arbitrary periodic dither. Our main result states that the averaged and the dithered system have qualitatively the same behavior when the nonlinearities have bounded variation and the dither has an absolutely continuous amplitude distribution function with bounded derivative and a sufficiently high frequency. The result appears to be fairly tight, because examples suggest that dithering might loose its effect when any of the assumptions are violated. The outline of the paper is as follows. The dithered system and its averaged counterpart are introduced in Section II. The main result on the approximation error between the dithered and the averaged systems is presented

The work by L. Iannelli and F. Vasca was supported by EC within the SICONOS project (IST2001-37172). The work by K. H. Johansson and U. Jönsson was supported the Swedish Research Council and by EC within the RECSYS project (IST-2001-32515).

L. Iannelli is with the Dept. of Computer and Systems Engineering, University of Napoli Federico II, 80125 Napoli, Italy, e-mail: luiannel@unina.it

K. H. Johansson is with the Dept. of Signals, Sensors \& Systems, Royal Institute of Technology, 10044 Stockholm, Sweden, e-mail: kallejes3.kth.se

U. Jönsson is with the Dept. of Mathematics, Royal Institute of Technology, 10044 Stockholm, Sweden, e-mail: ulfj@math.kth.se

F. Vasca is with the Dept. of Engineering, University of Sannio in Benevento, 82100 Benevento, Italy, e-mail: vasca@unisannio.it and proved in Section III. Some comments and remarks are finally given in Section IV.

\section{PRELIMINARIES}

The dithered and averaged systems are defined in this section, together with the amplitude distribution function of the dither signal.

\section{A. Dithered System}

The dithered feedback system is defined as

$$
\dot{x}=f_{0}(x, t)+\sum_{i=1}^{m} f_{i}(x, t) n_{i}\left(g_{i}(x, t)+\delta_{i}\right), \quad x(0)=x_{0} .
$$

The state $x$ belongs to $\mathbb{R}^{q}$. The functions $f_{i}: \mathbb{R}^{q} \times \mathbb{R} \rightarrow$ $\mathbb{R}^{q}, i=1, \ldots, m$, are assumed to be globally Lipschitz with respect to both $x$ and $t$, i.e., there exists a positive constant $L_{f}$ such that for all $x_{1}, x_{2} \in \mathbb{R}^{q}$ and $t_{1}, t_{2} \geq 0$,

$$
\left|f_{i}\left(x_{1}, t_{1}\right)-f_{i}\left(x_{2}, t_{2}\right)\right| \leq L_{f}\left(\left|x_{1}-x_{2}\right|+\left|t_{1}-t_{2}\right|\right) .
$$

We further assume that $f_{0}$ is piece-wise continuous in $t$, $f_{0}(0, t)=0, \forall t \geq 0$ and

$$
\left|f_{0}\left(x_{1}, t\right)-f_{0}\left(x_{2}, t\right)\right| \leq L_{f}\left|x_{1}-x_{2}\right|
$$

for all $x_{1}, x_{2} \in \mathbb{R}^{q}$ and $t \geq 0$. Similarly, the functions $g_{i}: \mathbb{R}^{q} \times$ $\mathbb{R} \rightarrow \mathbb{R}$ are assumed to have a common Lipschitz constant $L_{g}>0$, i.e.,

$$
\left|g_{i}\left(x_{1}, t_{1}\right)-g_{i}\left(x_{2}, t_{2}\right)\right| \leq L_{g}\left(\left|x_{1}-x_{2}\right|+\left|t_{1}-t_{2}\right|\right)
$$

for all $x_{1}, x_{2} \in \mathbb{R}^{q}, t_{1}, t_{2} \geq 0$. The nonlinearities $n_{i}: \mathbb{R} \rightarrow \mathbb{R}$ are assumed to be functions of bounded variation ${ }^{1}$. Hence, $n_{i}$ can be a discontinuous function, but it is necessarily bounded. Let $M_{n}:=\max _{i}\left(\left|n_{i}(0)\right|+T V\left(n_{i}\right)\right)$ denote such a bound, where $T V\left(n_{i}\right)$ is the total variation of $n_{i}$. Each dither signal $\delta_{i}:[0, \infty) \rightarrow \mathbb{R}$ is supposed to be a $p$-periodic measurable function bounded by $M_{\delta}$

When the differential equation (1) has a discontinuous right-hand side (due to that some $n_{i}$ are discontinuous), existence and uniqueness of solutions depend critically on the considered definition of solution [10]. In the following we assume that the differential equation (1) has at least one absolutely continuous solution $x\left(t, x_{0}\right)$ on $[0, \infty)$. Moreover, by using Lipschitz conditions on $f_{i}$, it is possible to show that there exists a positive constant $L_{x}$ such that $\left|x\left(t_{1}\right)-x\left(t_{2}\right)\right| \leq L_{x}\left|t_{1}-t_{2}\right|$ for almost all $0 \leq t_{1} \leq t_{2}<\infty$. Estimates of the Lipschitz constant $L_{x}$ can easily be obtained on any compact interval by using the assumptions above. Analogously it can be computed an upper bound on $f_{i}$ : $\left|f_{i}(x, t)\right| \leq M_{f}$ (see [11] for details).

\footnotetext{
${ }^{1}$ See [9] for an introduction to measure theory.
} 


\section{B. Amplitude Distribution Function}

Definition 2.1: The amplitude distribution function of a dither signal $\delta:[0, \infty) \rightarrow \mathbb{R}$ is the function $F_{\delta}: \mathbb{R} \rightarrow[0,1]$ defined as

$$
F_{\delta}(\xi)=\frac{1}{p} \mu\{t \in[0, p): \delta(t) \leq \xi\}
$$

where $\mu$ denotes the Lebesgue measure.

When the amplitude distribution function is absolutely continuous (with respect to its Lebesgue measure), the amplitude density function $f_{\delta}(\xi)$ is defined as

$$
f_{\delta}(\xi)=\frac{\mathrm{d} F_{\delta}}{\mathrm{d} \xi}(\xi) \text {. }
$$

The amplitude density and amplitude distribution functions play in a deterministic framework the same role as probability density and cumulative distribution functions play in a stochastic framework. In particular the amplitude distribution function is bounded, monotonously increasing, continuous from the right, and, if it is differentiable, its derivative obviously corresponds to the amplitude density function.

\section{Averaged System}

The averaged system is given by

$$
\dot{w}=f_{0}(w, t)+\sum_{i=1}^{m} f_{i}(w, t) N_{i}\left(g_{i}(w, t)\right), \quad w(0)=w_{0},
$$

where $N_{i}$ is called the averaged nonlinearity, ${ }^{2}$ and it is derived from the original nonlinearity $n_{i}$ and the dither signal $\delta_{i}$. We assume that there exists at least one absolutely continuous solution $w\left(t, w_{0}\right)$ defined on $[0, \infty)$. We will later show that this is the case when the amplitude distribution function is absolutely continuous and has a bounded derivative, because then the averaged nonlinearity is Lipschitz continuous.

The averaged nonlinearity is defined as follows ${ }^{3}$.

Definition 2.2: For a dither signal $\delta:[0, \infty) \rightarrow \mathbb{R}$ and a nonlinearity $n: \mathbb{R} \rightarrow \mathbb{R}$ the averaged nonlinearity $N: \mathbb{R} \rightarrow \mathbb{R}$ is defined as

$$
N(z) \triangleq \int_{\mathbb{R}} n(z+\xi) \mathrm{d} F_{\delta}(\xi)
$$

where the integral is a Lebesgue-Stieltjes integral.

In many cases the averaged nonlinearity can be formulated as a time average, as the following lemma states.

Lemma 2.1: [12], [13] The following equality holds provided that either side exists:

$$
N(z)=\frac{1}{p} \int_{[0, p)} n(z+\delta(s)) \mathrm{d} s .
$$

\footnotetext{
${ }^{2}$ In the literature, $N_{i}$ is sometimes called the smoothed nonlinearity, which however seems less appropriate here since $N_{i}$ can be a discontinuous function, as shown in Section IV.

${ }^{3}$ For the sake of simplicity from now on we will omit subscript $i$ when not necessary.
}

Two cases are of particular interest in the paper. First, when the amplitude distribution function is absolutely continuous, we have

$$
N(z)=\int_{\mathbb{R}} n(z+\xi) \mathrm{d} F_{\delta}(\xi)=\int_{\mathbb{R}} n(z+\xi) f_{\delta}(\xi) \mathrm{d} \xi,
$$

which is always well defined under the given assumptions on $n$.

Second, when the Lebesgue-Stieltjes measure corresponding to the amplitude distribution function has a decomposition (relative to the Lebesgue measure) into an absolutely continuous part and a singular part, we have (if $n$ is continuous at $z+\xi_{k}$ )

$$
\begin{aligned}
N(z) & =\int_{\mathbb{R}} n(z+\xi) \mathrm{d} F_{\delta}(\xi) \\
& =\int_{\mathbb{R}} n(z+\xi) f_{\delta}(\xi) d \xi+\sum_{k=1}^{Q} n\left(z+\xi_{k}\right) f_{k},
\end{aligned}
$$

where $f_{k} \neq 0$ are the jump discontinuities corresponding to the singular parts of the amplitude distribution function. Note that square wave and trapezoidal dither signals have this kind of amplitude distribution functions.

\section{Averaging Theorem}

The next theorem states conditions under which the averaged system approximates the behavior of the dithered system for a sufficiently high dither frequency. The theorem is stated for a finite time-horizon, but can be used to derive infinite horizon stability results, cf., [7].

Theorem 3.1: Consider the dithered system (1) and the averaged system (4) under the assumptions introduced in the previous section, i.e., for each $i=1, \ldots, m$,

(i) $f_{i}$ and $g_{i}$ are globally Lipschitz with Lipschitz constants, respectively, $L_{f}$ and $L_{g}$,

(ii) $f_{0}$ is globally Lipschitz with respect to $x$ with Lipschitz constant $L_{f}$, and $f_{0}(0, t)=0$,

(iii) $n_{i}$ is a function of bounded variation and sup-norm $\left\|n_{i}\right\|_{\infty} \leq M_{n}$

(iv) each dither $\delta_{i}$ is $p$-periodic with absolutely continuous amplitude distribution function $F_{\delta_{i}}$ and $L_{F} \triangleq$ $\sup _{\zeta \in \mathbb{R}}\left|f_{\delta_{i}}(\zeta)\right|<\infty$.

Then the averaged nonlinearities $N_{i}$ are Lipschitz continuous and the averaged system (4) has a unique absolutely continuous solution on $[0, \infty)$. Moreover, for any given $T>0$ and $x_{0} \in \mathbb{R}^{n}$, it holds that

$$
\left|x\left(t, x_{0}\right)-w\left(t, x_{0}\right)\right|=O(p), \quad \forall t \in[0, T] .
$$

The proof of the theorem is based on three lemmas. In the first lemma we show that the averaged nonlinearity under our assumptions is Lipschitz continuous, which implies that there exists a unique absolutely continuous solution of the averaged system on any finite time-horizon.

Lemma 3.1: Suppose $n$ is of bounded variation and that $F_{\delta}$ is absolutely continuous with $L_{F}=\sup _{\xi \in \mathbb{R}}\left|f_{\delta}(\xi)\right|<\infty$. Then

$$
N(z)=\int_{\mathbb{R}} n(z+\xi) f_{\delta}(\xi) d \xi
$$


is Lipschitz continuous with $L_{N} \leq L_{F} \cdot T V(n)$ and bounded with $\|N\|_{\infty} \leq\|n\|_{\infty}$.

Proof: We have

$$
\begin{aligned}
\mid N\left(z_{1}\right) & -N\left(z_{2}\right) \mid \\
& =\left|\int_{\mathbb{R}}\left[n\left(z_{1}+\xi\right)-n\left(z_{2}+\xi\right)\right] d F_{\delta}(\xi)\right| \\
& =\left|\int_{\mathbb{R}} n(\xi)\left[d F_{\delta}\left(\xi-z_{1}\right)-d F_{\delta}\left(\xi-z_{2}\right)\right]\right|
\end{aligned}
$$

Let $V(\xi)=F_{\delta}\left(\xi-z_{1}\right)-F_{\delta}\left(\xi-z_{2}\right)$. We have $V(\xi)=0$ for $\xi \notin S=\left[-M_{\delta}-\max \left(z_{1}, z_{2}\right), M_{\delta}-\min \left(z_{1}, z_{2}\right)\right]$. Hence, for any $I=[a, b] \supset S$ integration by parts gives

$$
\begin{aligned}
\mid N\left(z_{1}\right) & -N\left(z_{2}\right) \mid \\
& =\left|\int_{I} n(\xi)\left[d F_{\delta}\left(\xi-z_{1}\right)-d F_{\delta}\left(\xi-z_{2}\right)\right]\right| \\
& =\left|n(b) V(b)-n(a) V(a)-\int_{I} V(\xi) d n(\xi)\right| \\
& \leq \sup _{\xi \in I}|V(\xi)| \int_{I}|\operatorname{dn}(\xi)| \leq L_{F}\left|z_{1}-z_{2}\right| \cdot T V(n)
\end{aligned}
$$

where the last inequality follows because $V(a)=V(b)=0$ and

$$
|V(\xi)|=\left|\int_{z_{2}}^{z_{1}} f_{\delta}(\xi-\sigma) d \sigma\right| \leq L_{F}\left|z_{1}-z_{2}\right|
$$

The boundedness follows since

$$
|N(z)|=\left|\int_{\mathbb{R}} n(z+\xi) f_{\delta}(\xi) d \xi\right| \leq\|n\|_{\infty} \int_{\mathbb{R}} f_{\delta}(\xi) d \xi=\|n\|_{\infty}
$$

since $f_{\delta}$ is nonnegative.

It should be noticed that if $n$ is Lipschitz then the corresponding averaged nonlinearity $N$ will be Lipschitz independently on the characteristic of $F_{\delta}$, see [2]. Lemma 3.1 states that in order to have $N$ Lipschitz when $n$ is not Lipschitz, we need further assumptions on the dither. The next lemma is the key to the proof of Theorem 3.1.

Lemma 3.2: Suppose the signal $y:[0, p] \rightarrow \mathbb{R}$ has Lipschitz constant $L_{y}$. Introduce a constant $\tilde{y}$ satisfying

$$
\min _{s \in[0, p]} y(s) \leq \tilde{y} \leq \max _{s \in[0, p]} y(s) .
$$

Suppose that $F_{\delta}$ is absolutely continuous with $L_{F}=$ $\sup _{\xi \in \mathbb{R}}\left|f_{\delta}(\xi)\right|<\infty$. Then,

$$
\begin{aligned}
E & \triangleq\left|\int_{0}^{p} n(-y(s)+\delta(s)) \mathrm{d} s-\int_{0}^{p} n(-\tilde{y}+\delta(s)) \mathrm{d} s\right| \\
& \leq 2 L_{F} L_{y} T V(n) p^{2},
\end{aligned}
$$

where $T V(n)$ is the total variation of $n$.

Proof: From the previous definition of $F_{\delta}$ we introduce

$$
F_{-y+\delta}(\xi)=\frac{1}{p} \mu(\{s \in[0, p):-y(s)+\delta(s) \leq \xi\}),
$$

so that

$$
E=p\left|\int_{\mathbb{R}} n(\xi) \mathrm{d} F_{-y+\delta}(\xi)-\int_{\mathbb{R}} n(\xi) \mathrm{d} F_{-\tilde{y}+\delta}(\xi)\right| .
$$

By hypothesis

$$
\tilde{y}-L_{y} p \leq y(s) \leq \tilde{y}+L_{y} p, \quad \forall s \in[0, p]
$$

and thus it follows that for any $\xi \in \mathbb{R}$,

$$
F_{-\tilde{y}+\delta}\left(\xi-L_{y} p\right) \leq F_{-y+\delta}(\xi) \leq F_{-\tilde{y}+\delta}\left(\xi+L_{y} p\right) .
$$

On the other hand, since $F_{-\tilde{y}+\delta}$ is nondecreasing,

$$
F_{-\tilde{y}+\delta}\left(\xi-L_{y} p\right) \leq F_{-\tilde{y}+\delta}(\xi) \leq F_{-\tilde{y}+\delta}\left(\xi+L_{y} p\right) .
$$

By combining that (8) and (9) and using that $F_{-\tilde{y}+\delta}(\xi)=$ $F_{\delta}(\xi+\tilde{y})$ is Lipschitz and non-negative, we get

$$
\begin{aligned}
F_{-y+\delta}(\xi)-F_{-\tilde{y}+\delta}(\xi) & \leq F_{-\tilde{y}+\delta}\left(\xi+L_{y} p\right)-F_{-\tilde{y}+\delta}\left(\xi-L_{y} p\right) \\
& \leq 2 L_{F} L_{y} p .
\end{aligned}
$$

In an analogous way,

$$
-2 L_{F} L_{y} p \leq F_{-y+\delta}(\xi)-F_{-\tilde{y}+\delta}(\xi) .
$$

So we can write

$$
F_{-y+\delta}(\xi)=F_{-\tilde{y}+\delta}(\xi)+V(\xi)
$$

with $|V(\xi)| \leq 2 L_{F} L_{y} p$ and thus

$$
E=p\left|\int_{\mathbb{R}} n(\xi) \mathrm{d} V(\xi)\right| .
$$

Since for $s \in[0, p]$ we have $|y(s)-\tilde{y}| \leq L_{y} p$ and $|\delta(s)| \leq M_{\delta}$,

$$
V(\xi)=0, \quad \forall \xi \notin\left[-\tilde{y}-L_{y} p-M_{\delta},-\tilde{y}+L_{y} p+M_{\delta}\right] \triangleq S .
$$

The function $V(\xi)$ is of bounded variation and continuous from the right, since it is the difference of two functions that satisfy both these properties. By hypothesis $n$ is of bounded variation with total variation $T V(n)$ so we can integrate by parts [14]:

$$
\int_{[a, b]} n(\xi) \mathrm{d} V(\xi)=n(b) V(b)-n(a) V(a)-\int_{[a, b]} V(\xi) \mathrm{d} n(\xi),
$$

where right and left limits of $n$ and $V$ are used in order to cope with discontinuities. If $[a, b] \supset S$ then $V(a)=V(b)=0$, and thus

$$
E=p\left|\int_{S} V(\xi) \mathrm{d} n(\xi)\right| \leq 2 p^{2} L_{F} L_{y} T V(n),
$$

which proves the lemma.

Lemmas 3.1 and 3.2 are used to prove the following result (see [11] for the proof).

Lemma 3.3: If the assumptions of Theorem 3.1 hold, then there exist constants $\bar{K}, \tilde{K}>0$ such that

$$
\begin{aligned}
& \mid \int_{0}^{p} f_{i}(x, s) n_{i}\left(g_{i}(x, s)\right.\left.+\delta_{i}\right) \mathrm{d} s-\int_{0}^{p} f_{i}(w, s) N_{i}\left(g_{i}(w, s)\right) \mathrm{d} s \mid \\
& \leq \bar{K} \int_{0}^{p}|x(s)-w(s)| \mathrm{d} s+\tilde{K} p^{2}
\end{aligned}
$$

Now we can proceed by showing that the approximation error between the dithered and the averaged system can be arbitrarily small by increasing the dither frequency, as stated in the theorem. 
Proof of Theorem 3.1. Consider the dithered system (1) and the averaged system (4) on the time interval $[0, T]$ with $w_{0}=x_{0}$. By integrating the right-hand sides of (1) and (4), we can write

$$
\begin{aligned}
|x(t)-w(t)| \leq & \int_{0}^{t}\left|f_{0}(x, s)-f_{0}(w, s)\right| \mathrm{d} s \\
& +\sum_{i=1}^{m} \mid \int_{0}^{t}\left[f_{i}(x, s) \cdot n_{i}\left(g_{i}(x, s)+\delta_{i}\right)\right. \\
& \left.-f_{i}(w, s) \cdot N_{i}\left(g_{i}(w, s)\right)\right] \mathrm{d} s \mid
\end{aligned}
$$

for all $t \in[0, T]$.

If we introduce $l=\lfloor T / p\rfloor$, the largest integer such that $l p \leq T$, then by using the periodicity of $\delta_{i}$,

$$
\begin{aligned}
\mid x(t) & -w(t)\left|\leq \int_{0}^{t}\right| f_{0}(x(s), s)-f_{0}(w(s), s) \mid \mathrm{d} s \\
& +\sum_{k=0}^{l-1} \sum_{i=1}^{m} \mid \int_{k p}^{(k+1) p}\left[f_{i}(x(s), s) n_{i}\left(g_{i}(x(s), s)+\delta_{i}(s)\right)\right. \\
& \left.-f_{i}(w(s), s) N_{i}\left(g_{i}(w(s), s)\right)\right] \mathrm{d} s \mid \\
& +V_{1}(p), \quad \forall t \in[0, T],
\end{aligned}
$$

where the last term is bounded as

$$
\left|V_{1}(p)\right| \leq 2 m M_{f} M_{n} p
$$

The Lipschitz property of $f_{0}$ gives

$$
\begin{aligned}
\mid \int_{0}^{t}\left[f_{0}(x(s), s)\right. & \left.-f_{0}(w(s), s)\right] \mathrm{d} s \mid \\
& \leq L_{f} \int_{0}^{t}|x(s)-w(s)| \mathrm{d} s .
\end{aligned}
$$

Next we notice that each integral in the sum of (16) can be written

$$
\begin{aligned}
\int_{0}^{p}\left[f _ { i } ( x _ { k } ( s ) , s _ { k } ) n _ { i } \left(g_{i}\right.\right. & \left.\left(x_{k}(s), s_{k}\right)+\delta_{i}(s)\right) \\
& \left.-f_{i}\left(w_{k}(s), s_{k}\right) N_{i}\left(g_{i}\left(w_{k}(s), s_{k}\right)\right)\right] d s
\end{aligned}
$$

where the subscript $k$ denotes a time translation: $s_{k}=s+$ $k p, x_{k}(s)=x(s+k p)$ and similarly for $w$. Then applying Lemma 3.3, each integral

$$
\int_{0}^{p}\left(f_{i}\left(x_{k}, s_{k}\right) n_{i}\left(g_{i}\left(x_{k}, s_{k}\right)+\delta_{i}(s)\right)\right) \mathrm{d} s
$$

can be approximated by

$$
\int_{0}^{p} f_{i}\left(w_{k}, s_{k}\right) N_{i}\left(g_{i}\left(w_{k}, s_{k}\right)\right) \mathrm{d} s .
$$

Indeed, the Lipschitz assumptions on the $f_{i}$ and $g_{i}$ are uniform in $t$ so Lemma 3.3 can be applied to all functions $x_{k}$. The approximation error has an upper bound $\bar{K} \int_{0}^{p}\left|x_{k}-w_{k}\right| \mathrm{d} s+\tilde{K} p^{2}$. By summing all the contributions given by the time intervals $[k p,(k+1) p] \subset[0, T]$, we get

$$
\begin{aligned}
|x(t)-w(t)| \leq & K \int_{0}^{t}|x(s)-w(s)| \mathrm{d} s \\
& +m \tilde{K} p T+V_{1}(p)+V_{2}(p), \quad \forall t \in[0, T],
\end{aligned}
$$

where $K=L_{f}+m \bar{K}=L_{f}+m\left(M_{n} L_{f}+M_{f} L_{N} L_{g}\right)$ and $V_{2}(p)$ is bounded by

$$
\left|V_{2}(p)\right| \leq m \bar{K}\left(M_{x}+M_{w}\right) p,
$$

where $M_{x}$ and $M_{w}$ are easily derived upper bounds in $[0, T]$ of $x$ and $w$, respectively, see [11] for details.

By applying Grönvall-Bellman Lemma [15], the theorem follows since

$$
|x(t)-w(t)| \leq\left(m \tilde{K} T p+V_{1}(p)+V_{2}(p)\right) e^{K T} \quad \forall t \in[0, T]
$$

where the right hand side is of order $p$.

\section{DISCUSSION}

In this section we make some remarks on the assumptions and applications of Theorem 3.1.

\section{A. Smoothness of Amplitude Distribution Function}

The assumption on absolute continuity of the dither amplitude distribution function $F_{\delta_{i}}$ in Theorem 3.1 seems to be necessary (when $n_{i}$ is not Lipschitz continuous). Recall that for example a square wave violates this assumption. Indeed, an example in [7] shows that a dithered and an averaged relay system can have very different qualitative behaviors, when a square wave dither is used, even if the frequency of the dither is high. We showed in [8] that this phenomenon can be detected also in real applications. Next we provide a new example, which illustrates the importance of having dither signals with absolutely continuous amplitude distribution functions in nonsmooth systems.

Let us consider the dithered relay feedback system

$$
\dot{x}(t)=A x(t)+b \operatorname{sgn}(b x(t)+R+\delta(t)), \quad x(0)=x_{0},
$$

with

$$
\begin{gathered}
A=\left[\begin{array}{cc}
-1 & -1 \\
0 & -1
\end{array}\right], b=\left[\begin{array}{l}
0 \\
1
\end{array}\right], c=\left[\begin{array}{ll}
-1 & 0
\end{array}\right], x \\
\operatorname{sgn}(z)= \begin{cases}+1, & z>0 \\
0 & z=0 \\
-1, & z<0 .\end{cases}
\end{gathered}
$$

Let the external reference be constant $R=0.5$ and let $\delta(t)$ be a square wave dither of amplitude $M_{\delta}=0.5$, i.e.,

$$
R+\delta(t)= \begin{cases}1 & \bmod (t, p) \in\left[0, \frac{p}{2}\right) \\ 0 & \bmod (t, p) \in\left[\frac{p}{2}, p\right) .\end{cases}
$$

In this case the averaged system is equal to

$$
\dot{w}(t)=A w(t)+b N(c w(t)+R), \quad w(0)=w_{0}
$$

with

$$
N(z)= \begin{cases}-1, & z<-0.5 \\ -0.5 & z=-0.5 \\ 0, & |z|<0.5 \\ 0.5 & z=0.5 \\ +1, & z>0.5\end{cases}
$$


To argue about the evolution of the dithered and averaged systems, let us partition the state space into the following three regions and comment on the dynamics in each of them:

- Region $\Omega_{1}=\left\{x: x_{1}<0\right\}$ : In this region $n(c x+R+\delta)=$ 1 , so $\dot{x}=A x+b$ and the vector field of the dithered system coincides with the vector field of the averaged system. The equilibrium point of this system is given by $P_{1}=-A^{-1} b=(-1,1)^{T}$.

- Region $\Omega_{2}=\left\{x: x_{1}>1\right\}$. In this region $n(c x+R+\delta)=$ -1 , so $\dot{x}=A x-b$ and the dithered system coincides also here with the averaged system. The equilibrium point of the system is $P_{2}=A^{-1} b=(1,-1)^{T}$.

- Region $\Omega_{0}=\left\{x: 0<x_{1}<1\right\}$. In $\Omega_{0}$ the state does not affect the output of the relay, so the dithered system can be represented by the following linear system:

$$
\dot{\zeta}(t)=A \zeta(t)+b u(t),
$$

where $u$ is a periodic signal that switches between -1 (when $R+\delta(t)=0$ ) and +1 (when $R+\delta(t)=1$ ). The averaged system has an input equal to zero in this region, i.e., $\dot{w}(t)=A w(t)$. Note that if $w_{0} \in \Omega_{0}$, the solution $w(t)=\left(w_{1}(t), w_{2}(t)\right)^{T}$ is given by

$$
w_{1}(t)=e^{-t}\left[w_{1}(0)-t w_{2}(0)\right], \quad w_{2}(t)=e^{-t} w_{2}(0),
$$

as long as $0<w_{1}(t)<1$.

Now, consider solutions of the dithered and averaged systems as shown in Fig. 1. Let the initial conditions be equal $x(0)=w(0)$ and such that $x_{2}(0)=0$ and $0<x_{1}(0)<1$. Then, the averaged system has a solution that lies on the $x_{1}$ axis:

$$
w_{1}(t)=e^{-t} x_{1}(0), \quad w_{2}(t)=0 .
$$

Hence, the averaged system tends to the origin.

For the solution of the dithered system, note that in $\Omega_{0}^{+}=\left\{x \in \Omega_{0}: 0<x_{1}<1, x_{2}>1\right\}$ we have $\dot{x}_{1}<0$ and $\dot{x}_{2}<0$ while in $\Omega_{0}^{-}=\left\{x \in \Omega_{0}: 0<x_{1}<1, x_{2}<-1\right\}$ we have $\dot{x}_{1}>0$ and $\dot{x}_{2}>0$, cf., vector fields indicated in $\Omega_{0}^{-}$ and $\Omega_{0}^{+}$in Fig. 1. It then follows that the trajectory of the dithered system must leave $\Omega_{0} \backslash\left(\Omega_{0}^{+} \cup \Omega_{0}^{-}\right)$by crossing the line segment $\left\{x: x_{1}=0,0 \leq x_{2} \leq 1\right\}$. Moreover, in $\Omega_{0}$ the dithered solution can be represented as

$$
x(t)=e^{A t}\left(x(0)-\zeta_{0}\right)+\zeta_{s s}(t)
$$

where $\zeta_{s s}$ is the steady state $p$-periodic solution of (24) and

$$
\zeta_{0}=\left(I-e^{A p}\right)^{-1} \int_{0}^{p} e^{A(p-s)} b u(s) d s .
$$

Since $A$ is Hurwitz, $x(t)$ will converge to $\zeta_{s s}(t)$. It is possible to choose a sufficiently small dither period $p$ such that $\zeta_{s s}(t) \notin \Omega_{2}$, because $\zeta_{s s}(t) \rightarrow 0$ as $p \rightarrow 0$. It is thus clear that $x(t)$ eventually will cross the $x_{2}$ axis for some $0 \leq x_{2} \leq 1$. From Fig. 1, we conclude that the second orthant is an invariant set for the dynamics in region $\Omega_{1}$. Moreover, since the system matrix $A$ is Hurwitz, the dithered solution $x(t)$ will tend toward the equilibrium point $P_{1}$. Recall that the

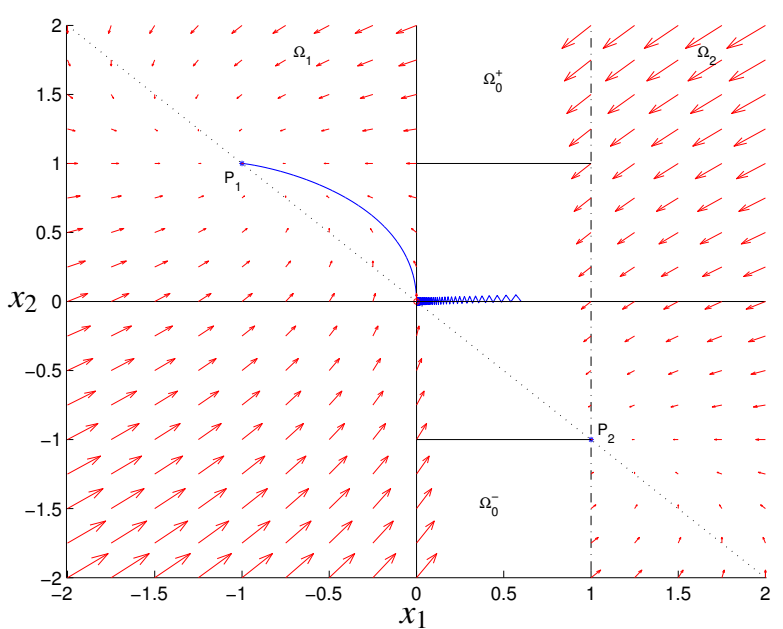

Fig. 1. Phase plane of a system with square wave dither. The indicated trajectory of the dithered system tends to the equilibrium point $P_{1}$. The corresponding trajectory of the averaged system tends to the origin. Hence, their characteristics are different.

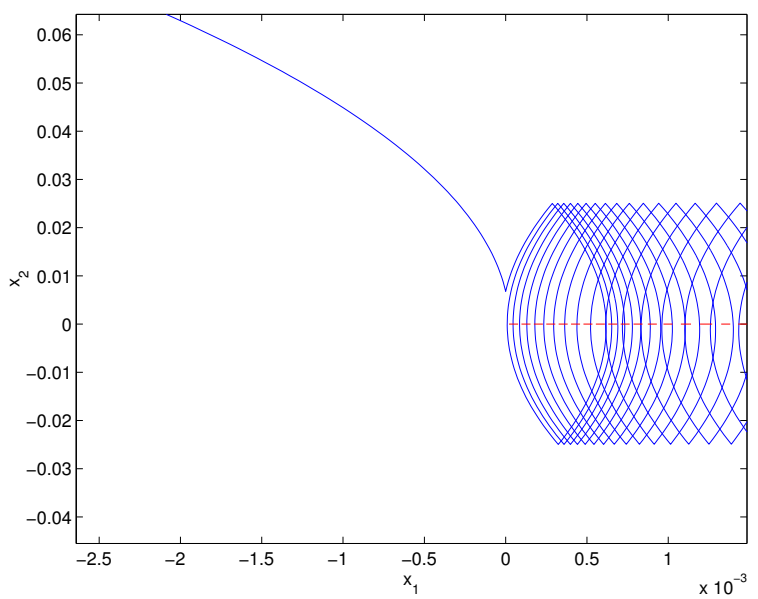

Fig. 2. Zoom of the phase plane in Fig. 1. Solutions of the dithered system (solid line) and averaged system (dashed line).

averaged system converges to the origin. The behaviors of the dithered system and the averaged system are hence quite different. See simulations in Fig. 2 and Fig. 3 for further comparison.

Even though we showed above that the dithered and the averaged system can behave qualitatively different and that this holds for any small dither period $p$, the example does not prove that the conclusion of Theorem 3.1 does not hold if the amplitude distribution function is not absolutely continuous. The reason for this is that it does not follow that the dithered state $x$ crosses the vertical axis $x_{1}=0$ in $[0, T]$. In particular, when increasing the dither frequency (as required by Theorem 3.1), the crossing is not guaranteed on a fixed interval $[0, T]$. The example does illustrate, however, how the dithered and the averaged solution bifurcate due to the fact that the averaged nonlinearity is discontinuous. We have discovered similar phenomena for limit cycles of the averaged and the dithered systems [7], [16], [17]. 


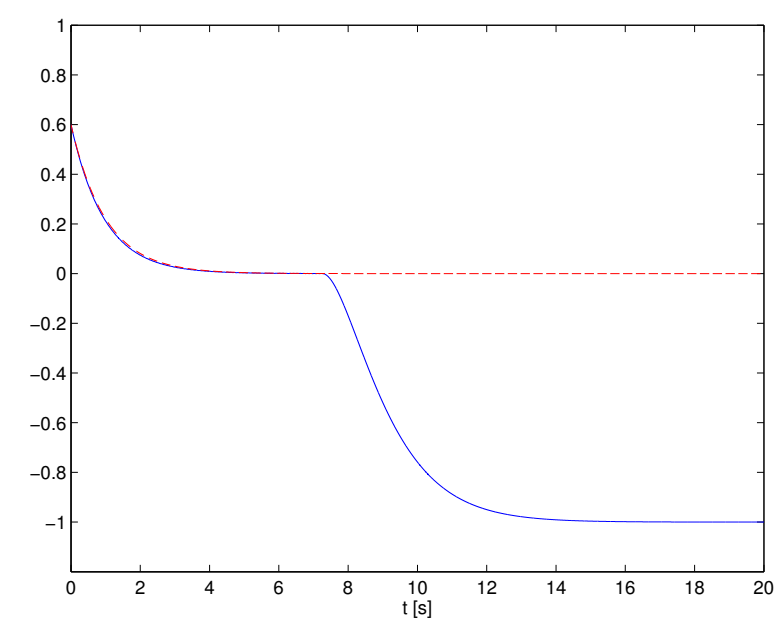

Fig. 3. Time evolution of $x_{1}$ (solid line) and $w_{1}$ (dashed line) of the dithered and averaged systems, respectively.

If assumption (iv) weakened such that the boundedness assumption on the density function is removed then the amplitude distribution function is no longer Lipschitz continuous and the conclusions of the theorem do not hold. For example, in [18] we have an example where an absolutely continuous dither with unbounded density function gives an averaged system that does not have a unique solution.

\section{B. Applications}

One of the main applications of Theorem 3.1 is to derive stability results for the dithered system. For example, if the averaged system is globally asymptotically stable, then it is possible to show that the dithered system is practically stable. Hence, a dither signal can be injected in order to (practically) stabilize a system. We refer to [7], [4], [19] for various results of this type.

In some applications it might be desirable to consider more general dither signals than periodic ones. It is possible to relax the assumption of Theorem 3.1 on the dither and consider so called $F$-repetitive dither as defined in [2]. Recall that a dither signal $\delta$ is $F$-repetitive if there exists an unbounded sequence $\left\{t_{k}\right\}, 0=t_{0}<t_{1} \ldots$, such that the maximal repetition $p=\sup _{k}\left(t_{k}-t_{k-1}\right)$ is bounded and the amplitude distribution function of $\delta$ on $\left(t_{k-1}, t_{k}\right)$, is equal to the amplitude distribution function on $\left(t_{0}, t_{1}\right)$.

Dither period, and amplitude and shape of the amplitude distribution function can be used as design variables. Theorem 3.1 shows that the dither period determines the approximation between the dithered and averaged systems. Moreover, for desired performance of the dithered system, since each amplitude distribution function defines a class of dither signals which gives rise to the same averaged nonlinearity, it is more appropriate to design the amplitude and the shape of the amplitude distribution function instead of looking at the dither amplitude and shape. For instance, the shape of the amplitude distribution function can be critical for determining a compromise between the maximal reduction of the nonlinear sector and the local attractivity of an equilibrium. We will discuss this further in our future work.

\section{CONCLUSIONS}

We have proved that dither can be efficiently used for the averaging of a quite general class of nonsmooth nonlinear systems, provided that the amplitude distribution function of the dither is Lipschitz. This condition, which is not needed for smooth Lipschitz nonlinear systems, suggests that the dither shape must be carefully chosen for smoothing common discontinuous systems such as mechanical systems with friction, power electronic converters and other hybrid systems.

\section{REFERENCES}

[1] S. Mossaheb, "Application of a method of averaging to the study of dither in non-linear systems," International Journal of Control, vol. 38, no. 3, pp. 557-576, September 1983.

[2] G. Zames and N. A. Shneydor, "Dither in non-linear systems," IEEE Transactions on Automatic Control, vol. 21, no. 5, pp. 660-667, October 1976.

[3] — - "Structural stabilization and quenching by dither in non-linear systems," IEEE Transactions on Automatic Control, vol. 22, no. 3, pp. 352-361, June 1977.

[4] A. K. Gelig and A. Churilov, Stability and Oscillations of Nonlinear Pulse Modulated Systems. Berlin: Birkhäuser, 1998.

[5] A. Teel, L. Moreau, and D. Nesic, "Input-to-state set stability of pulse width modulated systems with disturbances," Systems and Control Letters, vol. 51, January 2004.

[6] B. Lehman and R. Bass, "Extensions of averaging theory for power electronics systems," IEEE Transactions on Power Electronics, vol. 11 , no. 4, pp. 542-553, 1996.

[7] L. Iannelli, K. Johansson, U. Jönsson, and F. Vasca, "Dither for smoothing relay feedback systems: an averaging approach," IEEE Transactions on Circuits and Systems, Part I, vol. 50, no. 8, pp. 1025-1035, August 2003.

[8] - "Practical stability and limit cycles of dithered relay feedback systems," in Proc. of IEE European Control Conference, Cambridge, UK, September 2003.

[9] R. Wheeden and A. Zygmund, Measure and Integral. New York, NY, USA: Marcel Deeker Inc., 1977.

[10] A. F. Filippov, Differential Equations with Discontinuous Righthand Sides. Kluwer Academic Publishers, 1988.

[11] L. Iannelli, K. Johansson, U. Jönsson, and F. Vasca, "Conditions on the dither shape in the averaging of switched systems," Department of Mathematics, Royal Institute of Technology, Tech. Rep. TRITA/MAT-03-OS08, September 2003.

[12] K. L. Chung, A Course in Probability Theory. Orlando, FL, USA: Academic Press Inc., 1974.

[13] S. Taylor, Introduction to Measure and Integration. Cambridge University Press, 1966.

[14] Riesz and Sz-Nagy, Functional Analysis. Dover Pubns, 1990.

[15] S. Sastry, Nonlinear Systems: Analysis, Stability and Control. New York: Springer-Verlag, 1999.

[16] L. Iannelli, "Dither for smoothing relay feedback systems: an averaging approach," Ph.D. dissertation, University of Napoli "Federico II", Napoli, Italy, Nov. 2002, http://cds.unina.it/ luiannel/eng/public.htm.

[17] L. Iannelli, K. Johansson, U. Jönsson, and F. Vasca, "Effects of dither shapes in nonsmooth feedback systems: Experimental results and theoretical insight," in Proc. of IEEE Conference on Decision and Control, Maui, Hawaii (USA), December 2003.

[18] L. Iannelli, K. H. Johansson, U. Jönsson, and F. Vasca, "Averaging of nonsmooth systems through dithered switchings," 2004, in preparation.

[19] L. Moreau and D. Aeyels, "Practical stability and stabilization," IEEE Trans. Automat. Control, vol. 45, no. 8, pp. 1554-1558, august 2000. 